\title{
Seoul: Eindrücke einer Reise Der diesjährige 22. Dermatologische Weltkongress
}

\author{
Impressions from Seoul: The 22nd World Congress of Dermatology
}

Autor

Institut

\section{E. Orfanos}

Ehem. Direktor der Klinik und Poliklinik für Dermatologie, Universitätsklinikum Benjamin Franklin der FU Berlin
Bibliografie

Dol http://dx.doi.org/

10.1055/s-0030-1256726

Akt Dermatol 2011; 37:

312-316 @ Georg Thieme

Verlag KG Stuttgart · New York

ISSN 0340-2541

Korrespondenzadresse

Prof. Dr. med. Dr. h.c. mult.

Constantin E. Orfanos

Sybelstr. 37

10629 Berlin

constantin.orfanos@charite.de
Aus früheren Reisen war mir Korea in bester Erinnerung geblieben. Vermutlich lag es auch daran, dass meine Frau und ich früher das Privileg hatten, in Begleitung koreanischer Kollegen, die uns mit viel Umsicht und Sachkenntnis führten, ausgesucht schöne Seiten Koreas kennenzulernen. Seoul selbst schien uns dabei weniger attraktiv, nach Ableistung meiner Vortragsverpflichtungen verließen wir die Stadt und bereisten mit großer Begeisterung die Provinz. Nicht zuletzt der Süden der Halbinsel mit Gyeongju, der alten Kapitale aus der Blüte der Silla-Dynastie während des ersten Jahrtausends unserer Zeitrechung, hatte uns tief beeindruckt.

Gyeongju, heute eine kleine Provinzstadt, ist wie ein offenes Landschafts-Museum. Es ist ein Welterbe der UNESCO, in dem die zahlreichen Kulturdenkmäler einschließlich der bis zu 20 Meter hohen, begrünten Tumuli-Gräber der Könige und Strategen des vereinigten Silla-Reichs bis an die Grenze der heute bewohnten Siedlungen reichen. Von jugendlicher Neugierde angespornt, war es für uns ein Vergnügen, tagelang die mühsamen Wege zu den verschiedenen Pagoden zu laufen und die kleinen Buddha-Tempel aufzusuchen, die in den Hügeln der Umgebung in Grotten versteckt sind, umgeben von dichten Gingo-Bewaldungen in herrlichen herbstlichen Farben. Im Süden der Halbinsel hatten wir das Land des koreanischen Buddha kennengelernt, an manchen Stellen eine nahezu mystische Erfahrung; als solche blieb sie jedenfalls in meiner Erinnerung haften. Im reich bestückten Museum von Gyeongju hatten wir die kunstvollen Grabbeigaben aus den wenigen geöffneten Königsgräbern bewundert, die anderen Grabstätten wurden respektvoll gepflegt und blieben verschlossen, um ihre Geheimnisse für kommende Generationen zu bewahren. Bei anderen Reisen standen alte buddhistische Klöster im Norden und Feste mit Tanzvorführungen hübscher Koreanerinnen in ihren traditionellen bunten Kleidern auf unserem Programm, ebenso wie private Kunstsammlungen und Künstlerwerkstätten, wo die atemberaubend ästhetische koreanische Celadon-Keramik nach alten Rezepten noch hergestellt wurde.

Insgesamt war es das traditionelle Bild Koreas, das wir als kostbare Erinnerung behalten hatten und später zu Hause auf diverse Weise pflegten, das zusammen mit der köstlichen Küche und der Trinkfreudigkeit der Koreaner unsere als kurz empfundenen Aufenthalte in diesem ostasiatischen Land zu einem Erlebnis gemacht hatten.

Bei meinem letzten Besuch anlässlich des 22. Dermatologischen Weltkongresses war Seoul mein alleiniges Reiseziel. Nach der Landung in Incheon ging es über den „Olympic Highway“ direkt in die $70 \mathrm{~km}$ weit entfernte koreanische Hauptstadt, die inzwischen mächtig gewachsen ist. Groß-Seoul soll über 20 Millionen Einwohner haben, manche Koreaner erzählen gern, dass sie das größte oder zweitgrößte Ballungszentrum der Welt sei, sie strahlt stellenweise den „westlichen“ Fortschritt mit all seinen superglatten Facetten aus, die in vielen Großstädten unserer Welt zu finden sind. Korea reiht sich heute in die Riege der ostasiatischen „Tigerstaaten“ ein, diesmal unter der Führung des wieder erstarkten chinesischen Nachbarn.

\section{Das Umfeld}

$\nabla$

Der Kongress fand im relativ neuen Wirtschaftszentrum der Stadt im Süden von Seoul statt. Das hochmoderne Kongressgebäude, das gleichzeitig als Geschäftszentrum aufgestellt ist, wird von breiten Straßenzügen mit Hochbauten und Apartmentblocks aus Stahl und Glas umsäumt, eine Etage tiefer befinden sich die in Japan und Korea so beliebten, labyrinthisch verzweigten malls, in denen bis in die späten Abendstunden dichte Massen von Menschen herumlaufen, essen oder sich mit Leidenschaft dem Konsum widmen. Die 
Vielzahl der kleinen und größeren Geschäfte mit den vielfältigen bunten Attributen und diversen Accessoires westlicher Mode, für die man neuerdings erstaunlich hohe Preise verlangt, war verwirrend. Dazwischen hielten Kaufläden aller Art, Elektronikund Automatengeschäfte, Friseure, Massage- und Kosmetiksalons, Kitas, „dental clinics“ und ähnliche Geschäfte und Einrichtungen ihre Türen offen, überall wurde Kleinkram angeboten, um die Touristen zu locken. Lediglich die Küchengerüche der unzähligen kleinen koreanischen Restaurants, Bars und Imbissbuden, die an jeder Ecke auftauchten, erinnerten den Besucher daran, dass er sich in Ostasien befand.

Gerade in diesen malls konnte man den Eindruck nicht loswerden, dass Korea nun endgültig dabei ist mit seinen alten Traditionen zu brechen und in globalisierter Flachheit seinen Charme zu verlieren. Der Anblick der Massen modisch gekleideter junger Menschen, die in ihrer Freizeit sorglos herumschlenderten und sich mit dem größten Eifer und Hingabe einem Allerwelts-Konsum widmeten, war für mich bedrückend. Es war eine Szenerie, die mich teilweise an Zukunftsvisionen von Isaac Asimov erinnerte.

\section{Der Kongress}

$\nabla$

Der Schock durch den medizinischen Weltkongress, dessen Besuch Anlass meiner Reise gewesen ist, war nicht kleiner: Die Eröffnung wurde von einer Multimedia-Show einer gewaltigen Drumband mit den obligatorischen Riesentrommeln begleitet, die in ihrer Lautstärke und unkontrollierter postmoderner Wildheit nichts zu wünschen übrig ließ. Die Kongressteilnehmer schienen überwältigt, zumal die schrillen Megalaute aus den zahlreichen Lautsprechern, die im Saal gleichmäßig verteilt waren, einem die Trommelfelle sprengten. In den kurzen Pausen zwischen den Darbietungen langhaariger wilder Trommler und akrobatischer Tänzer, die auf der langen Bühne in der raschesten Abfolge, die man sich vorstellen konnte, ihre Künste vorführten, informierte uns die hübsche Ansagerin mit freundlichem Lächeln, dass dieses Spektakel die Eröffnung eines medizinischen Weltkongresses war, um uns dann im gleichen Atemzug darauf aufmerksam zu machen, dass sie ihr Kleid einem berühmten westlichen Designer verdanke, ich glaube aus Hamburg, obwohl ich mir darüber nicht sicher bin, da ihr Pigeon-English mit koreanischem Akzent als vereinfachte Form der Kommunikation nicht immer verständlich war. Ob wir ihr Kleid schön fanden, wollte sie jedenfalls wissen, worauf sie in betont lässiger Art westlicher Showmaster uns um kräftigen Applaus bat, den sie von vielen faszinierten Zuschauern, wenn auch etwas zögernd, bekam.

Um mich herum konnte man im Dunkeln die Gesichter der vielen Kollegen nicht sehen, geschweige denn ihre Gedanken ablesen. Schauelemente waren auch früher in den Eröffnungsveranstaltungen von Weltkongressen vorhanden, aber dies übertraf alle meine Erwartungen. Ich dachte an frühere Erfahrungen in Korea, Tagungen, die ich in einem reizvoll traditionellen koreanischen Ambiente erlebt hatte, und wollte den Riesensaal möglichst unauffällig verlassen, ließ es mir jedoch nicht nehmen, die Ansprache des Präsidenten der Internationalen Liga, Prof. JeanHilaire Saurat, zu hören, die allerdings nur wenige Minuten dauern durfte. So wollte es das „Protokoll“, sagte er mir später. Im Schutz der Dunkelheit flüchtete ich in die hintersten Reihen des Saales und harrte auf das Ende. Nach einigen weiteren Grußworten und Darbietungen brachte die hübsche Showmasterin das Ganze erfolgreich zum Abschluss, indem sie ein 10-minütiges Schlusswort dem Vertreter einer bekannten Kosmetikfirma über- ließ. Seine Hinweise auf die Leistungen der koreanischen Kosmetikindustrie waren vermutlich als Höhepunkt der Veranstaltung gedacht. Ich spürte Schmerzen in den Ohren und hatte das Glück, dass die Eröffnungszeremonie zu einem baldigen Ende kam.

Viele medizinische Großkongresse scheinen sich inzwischen zu derartigen Massenveranstaltungen entwickelt zu haben, erfuhr ich in späteren Gesprächen mit meinen jüngeren Kollegen. Sie sind endgültig dabei, ihren ursprünglichen Ansatz eines hoch angesiedelten wissenschaftlichen Ereignisses aufzugeben. Korea durfte hier keine Ausnahme machen. Bei den Vorträgen der wissenschaftlichen Sitzungen, die ich später besuchte, war mein persönlicher Eindruck, dass allenfalls Weiterbildunginhalte noch präsentiert bzw. aufrechterhalten wurden und eine gewisse Beachtung fanden, während die überwiegende Mehrheit der Teilnehmer eher an den Veranstaltungen und Pröbchen der ausstellenden Industrie interessiert war. Ich selbst halte jedenfalls als Zuhörer wenig von solchen Veranstaltungen, wenn ich die Chance außer Acht lasse, Freunde aus allen Ecken dieser Welt dort anzutreffen.

Die Redner waren auch, mit wenigen Ausnahmen, nicht ganz unschuldig an dieser unglücklichen Entwicklung. Viele erfahrene Forscher passten sich dem allgemeinen Trend an und drückten ihrerseits das Niveau ihrer Ausführungen nach unten, waren bemüht, gedankliche Feinheiten auszulassen, und beschränkten sich auf die wesentlichen Punkte ihrer Aussagen, um bei ihren Zuhörern besser anzukommen. Ein Problem schien mir dabei die Sprache zu sein, denn mit dem Kongress in Korea wurden die üblichen fünf offiziellen Sprachen unserer früheren Weltkongresse endgültig aufgegeben, zugunsten eines Einheits-Englisch mittlerer oder gar schlechter Qualität, je nach Vermögen des Redners. Was für ein Jammer! Vorbei die Zeiten, in denen man prominente Vertreter unseres Faches in ihrer eigenen Sprache hören und ihre persönliche Ausstrahlung am Rednerpult spüren konnte, während man komplizierte Inhalte über die simultane Übersetzung klar und deutlich vermittelt bekam. Kosten für Simultanübersetzer müssten heute aus finanziellen Überlegungen „eingespart" werden, wurde mir gesagt, darüber hinaus würde Englisch als einheitliche Vortragssprache der besseren Kommunikation dienen. Das Argument ist nur scheinbar schlüssig: Bei der persönlichen Kommunikation könnte man ja ohnehin das übliche Englisch verwenden, bei den wissenschaftlichen Präsentationen hingegen geht durch die oft unzulängliche Sprachenbeherrschung inhaltlich viel Wertvolles verloren oder wird gar nicht angeführt. Auch auf die Chance, die Besonderheiten und Feinheiten unserer multikulturellen Welt während der alle fünf Jahre stattfindenden Weltkongresse zu pflegen, wird verzichtet, ihr Charme wird dem Geldwahn geopfert. Der Einzug der Ökonomie in Bildung und Wissenschaft ist allgegenwärtig, der Wille zum besseren Verständnis des anderen wird peu á peu aufgegeben.

Dazu kommt, dass ein immer größer werdender Teil der Vortragenden bei den heutigen Kongressen finanziell, direkt oder indirekt, von Industriefirmen unterstützt wird, sodass vielfach gefällige Vorträge im Sinne der Sponsoren gehalten werden. Beraterverträge ermöglichen Honorare, Übernahme von Reisekosten oder sonstige Vergünstigungen, einen Eindruck davon kann man aus den Erklärungen am Ende des gewichtigen Programmheftes entnehmen. Es dürfte inzwischen ein offenes Geheimnis sein, dass die Inhalte mancher Kongress-Präsentationen von den Marketing-Spezialisten der Industrie beeinflusst oder zumindest feinfühlig durch sie gesteuert werden. Vielfach werden die Dias und die Kernaussagen vorgefertigt, der Sprecher beschränkt sich 
weitgehend in der Präsentation auf das Gewünschte. Von den meisten Plenarvorträgen abgesehen, war bei einigen Beiträgen des Hauptprogramms die industrielle Liaison deutlich, bei anderen war für den weniger kundigen Zuhörer die Verbindung weniger gut erkennbar. In den Marketing-Sitzungen der Industrie, die man immer noch euphemistisch als „Satelliten-Symposia“ deklariert, wurden die Beiträge gar reichlich mit Showelementen geschmückt und mit kleineren Geschenken, Buffets etc. begleitet, um die „Kunden“ zu locken.

In den überdimensionierten Hallen der begleitenden Ausstellung standen vor den diversen Ständen lange Schlangen von Teilnehmern, deren offensichtliches Bemühen darin bestand, große Taschen mit möglichst vielen Musterproben und Pröbchen der Aussteller zu füllen. Was für ein Anblick! Wie konnten sich Ärzte und Ärztinnen so verhalten? Die mit Schminken, Shampoos und sonstigen Mustertuben prall gefüllten, großen Plastiktaschen ließen den Eindruck einer Markthalle aufkommen. Gleichzeitig waren die Vortragssäle, in denen die Sitzungen der eigentlichen Dermatologie stattfanden, fast leer, den meisten Kongressbesuchern ging es offenbar nicht mehr um Inhalte. War dies meine akademische Jungfamilie? Ich fühlte mich betroffen, es war fast würdelos.

Beim Rückflug hatte ich Gelegenheit mit der klugen Repräsentantin einer seriösen pharmazeutischen Firma ein längeres Gespräch über ihre Kongresseindrücke zu führen. Auch sie sei vom Verhalten meiner Kollegen und Jungakademiker schockiert, der Anblick all dieser jungen Ärzte ..., alles studierte Menschen, zum Teil gestandene Akademiker..., in diesen Schlangen..., das war nicht schön, meinte sie. Hätten sie es nötig? Natürlich sei die Kosmetik-Industrie dafür verantwortlich, man lockt doch die Massen von Teilnehmern mit vielen Geschenken, Verlosungen teurer iPads, Einladungen zum Essen und anderen Dingen regelrecht an, während Diskussion und Gedankenaustausch an den Ständen der pharmazeutischen Industrie kaum noch stattfinden. Der deutsche, strenge „Pharma-Codex“, den alle Hersteller verschreibungspflichtiger Medikamente einhalten müssen, verbiete solche Dinge. Zweifellos, die Verflechtung der medizinisch-kosmetischen Industrie mit der Medizin hat inzwischen Ausmaße erreicht, die für viele ärgerlich sind. Meine Gesprächspartnerin schien mir jedenfalls enttäuscht und stellte für sich die Frage, ob sich der ganze Aufwand für ihre solide pharmazeutische Firma gelohnt hätte.

In der Tat, die Stände der Kosmetikahersteller waren während dieses Weltkongresses gezielt und ständig belagert und die der Laser und ähnlicher Geräte gut besucht, demgegenüber war an den Ständen der Pharmafirmen der Andrang bescheiden, man beschränkte sich auf ein Erfrischungsgetränk und gelegentliches Plaudern. Ich dachte zurück an Zeiten, in denen das Auftreten der Pharmavertreter bescheiden war und wir zumindest teilweise das Gefühl hatten, dass bei unseren Begegnungen, trotz der Verkaufsargumente, immerhin der Patient im Vordergrund stand. Man müsste vielleicht ein gewisses Verständnis für unsere jungen Ärzte haben: Wenn bürgerliche Tugenden in der Gesellschaft nicht mehr gefragt wären, warum sollten die sogenannten „akademischen“ weiter Beachtung finden? Wie auch immer, die Entscheidung des BGH, ob praktizierende Ärzte der Korruption belangt werden können, erwarte ich mit Spannung, ein klares Wort darüber ist längst fällig.

Ein unabhängiger, neutraler Beobachter muss jedoch die Frage stellen, ob die Dermatologie weiterhin solche Weltkongresse braucht? Und wenn ja, ist Weiterbildung das Ziel oder das globale
Geschäft? Wissenschaftliche Ergebnisse seriöser Forscher werden heute nahezu ausschließlich in den einschlägigen wissenschaftlichen Journalen veröffentlicht oder in anderen hochwertigen, meist kleineren Veranstaltungen präsentiert, wo die Spezialisten unter sich sind. Weltkongresse seien dafür nicht der richtige Platz, gäbe es zu bedenken. Andererseits, das kritische Abfiltrieren und sorgfältige Abwägen, das Formulieren von Zielsetzungen, Dinge, die bisher alle fünf Jahre Aufgabe der Weltkongresse waren und in den Vorträgen der besten Kliniker und Forscher weltweit als Resümee und Standortbestimmung des Faches galten, bleiben auf der Strecke. Eine Standortbestimmung findet durchweg nicht statt, eine Richtschnur für die Zukunft brauche man nicht, meint man. Die Resümees werden dem Einzelnen überlassen, die Vielfalt der Interessen führt aber dazu, dass die Rat- oder Orientierungslosigkeit zunimmt. Es entstehen beliebige, teilweise unkontrollierte Trends, denen man kritiklos oder vorteilsorientiert nach dem Motto „everything goes“ nachläuft, Hauptsache, das „Geschäft“ läuft. Kann dies das Ziel der Dermatologie als Heilkunst und Wissenschaft sein?

Großkongresse scheinen heute nur noch Schauplätze für Mediziner zu sein, die in ihrer überwiegenden Mehrheit an den finanziellen Strippen der pharmazeutischen und kosmetischen Industrie hängen, als ob sie am Hungertuch nagen würden und keine andere Einnahmequelle hätten. Man braucht sich nicht zu wundern, mit dem Einzug der Ökonomie in der modernen Medizin wurden bei den Ärzten die Berührungshemmungen zum industriellen, profitorientierten Denken abgebaut, die monetären Interessen geben die Richtung vor, der wissenschaftliche Wert der Kongresse nähert sich dem Nullpunkt.

Nirgendwo konnte dies in Bezug auf die Dermatologie besser erkennbar werden als im ostasiatischen Raum, wo die weibliche Schönheit hoch im Kurs steht und die Geschäfte der Kosmetikindustrie boomen.

\section{Tempel, Königsgräber, Museen}

$\nabla$

Eine gute Abwechselung vom Kongressprogramm war Bongeumsa, der älteste buddhistische Tempel Seouls aus der Silla-Periode, den man auf einer grünen Anhöhe in der Nähe des Kongresszentrums leicht zu Fuß erreichen konnte. Dieses Tempelareal und die Seonjeonneung-Königsgräber aus der Joseon-Periode im 16. Jahrhundert sind die einzigen Sehenswürdigkeiten im Süden der Hauptstadt. Bongeumsa geht auf das 8. oder 9. Jahrhundert zurück und gehört zur traditionstreuen Jogye-Sekte, deren Tempel in einer weitläufigen grünen Anlage gut erhalten und für die Besucher offen sind. Er ist einer der Anlaufpunkte der landesweiten Initiative „stay in temple“, die es dem Besucher ermöglicht, einige Tage in den Unterkünften der Mönche zu verbringen und an ihrem Tagesablauf aktiv teilzunehmen. Das Programm hat in den letzten Jahren sowohl unter Koreanern als auch unter westlichen Besuchern Zuspruch gefunden, wobei das Motto der JogyeAnhänger

\section{„Bongeumsa, be One through Practice and Effort“ in etwa: \\ „Bongeumsa, sei Einzig (Du selbst) durch praktische Übung und Mühe“}

an die puristische Tradition des koreanischen Buddhismus erinnert und nicht wenige anspricht. Ich weiß nicht, wie lang man 
sich vorher anmelden muss oder wie umständlich die Formalitäten für derartige Unternehmungen sind, mir schoss schnell der Gedanke durch den Kopf, aus meinem glattpolierten Hotel auszuziehen und einige Tage im nahe gelegenen Tempel zu verbringen. Leider habe ich nicht den Mut dazu aufbringen können, doch einige ruhige Nachmittagsstunden in Bongeumsa haben mir geholfen, meine Unzufriedenheit zu überwinden. Die laufenden Zeremonien mit den monotonen Gesängen und die ausgeglichenen Gesichter der Betenden strahlten Ruhe und Zuversicht aus. Ein besondere Sehenswürdigkeit Seouls ist das Leeung- oder Samsung-Museum, von der gleichnamigen Firma konzipiert und unterhalten, das in zwei supermodernen Bauten europäischer Architekten zum einen ausgewählte Objekte traditioneller koreanischer Art, vor allem Keramik und Metallarbeiten, und zum anderen Werke zeitgenössischer Künstler wie Beuys, Rothko, Francis Bacon, Koons, natürlich auch des Koreaners Nam June Paik, bis zu neuesten Werken von Andreas Gursky und Damiel Hirst zeigt. Das Ganze besticht durch die hohe Qualität der ausgesuchten und ausgestellten Objekte und den Charakter eines privaten exklusiven Museums, doch der Versuch das Alte mit dem Neuen zu verbinden gelingt nicht, die Welten, die sie trennen, werden vielmehr nüchtern und brückenlos aufgezeigt. Spätestens beim Anblick der Riesenspinne, einer surrealistischen Skulptur von Louise Bourgeois, in der vorgelagerten Terrasse kommt einem der Gedanke, ob alles Gezeigte überhaupt zur gleichen Gattung unter dem Begriff „Kunst“ einzuordnen ist.

Einen ganzen Tag widmete ich dem Nationalmuseum, das sich im Norden der koreanischen Hauptstadt, in der Nähe der früheren Altstadt, befindet. Ich war voller Erwartung, da ich das alte Nationalmuseum Seouls, das in einer alten Palastanlage untergebracht war, noch in prächtiger Erinnerung hatte. Meine Eindrücke aus dem neuen Gesamtkomplex blieben diesmal zwiespältig: Das neue Nationalmuseum erschien mir wie ein megalomaner Bau, imposant in seinen Zementdimensionen, doch ohne architektonische Rücksicht auf die traditionelle Feinheit und den meditativen Charme der Kunst, die man dort präsentiert. Immerhin war ich dankbar, mich einen weiteren halben Tag von den vielfältigen Ergebnissen des Fortschritts der modernen Medizin fernzuhalten. Ich fand viel Wissenswertes über die Historie, die Epochen des vereinten Silla-Reichs im 7. bis 9. Jahrhundert, der späteren Goryeo- und der anschließenden Joseon-Periode gingen in den leicht verdunkelten Sälen an einem vorbei, dazu einiges aus Zentral- und Südostasien, der nördlichen Seidenstraße von $\mathrm{Bu}$ chara über Kashgar und Turfan (Bizeklik) bis nach Chang'an und von dort nach Gyeongju im Süden der koreanischen Halbinsel. Die besten Exponate, die feinsinnigen kalligrafischen Blätter, die wunderbaren Exemplare der klassischen Celadon-Ware aus der Goryeo-Zeit (937-1392) und des weißen koreanischen Porzellans (Baekja genannt) aus der Joseon-Periode (1392-1910) waren in den riesigen, verwinkelten Sälen des neuen Museums ästhetisch durchdacht aufgestellt, man konnte alles um sich herum verdrängen und den Anblick genießen. Vor allem die koreanische Keramikkunst ist einer der Glanzpunkte Ostasiens. Lediglich die laut schreienden Kinderklassen, die von ihren überforderten Lehrern durch die Säle und Hallen des Museums in schnellem Schritt geführt, man könnte fast sagen gejagt, wurden, machten zuweilen einen ruhigen Museumsbesuch unmöglich. Im weiten grünen Gartenareal des Gesamtkomplexes sind mehrere Bauten, darunter auch eine Bibliothek, ein Bildungszentrum und auch ein Kindermuseum untergebracht, doch der Aktivitätsdrang der kleinen quirligen Geister machte vor nichts Halt, ohne
Hemmungen liefen sie in wechselnden Scharen durch die Säle und taten ihre Freude kund, den Tag außerhalb ihrer Klassenräume zu verbringen. Immerhin, der Anblick der laut schreienden Kinder, die um einen herum rannten und kicherten, war mir keinesfalls unangenehm, sie rauschten an einem vorbei, ihre hübschen runden Gesichter betrachteten mich offen und neugierig, ohne auch nur anstandshalber die wertvollen Exponate in den Vitrinen eines Blickes zu würdigen. Manche wagten ein flüchtiges „hallo“ oder „how are you?“, stolz, ihr Englisch zu üben, das ich gern beantwortete. Sie erinnerten mich an die ausgelassenen Kinder Ostafrikas, die kleinen „totos“, die meine Frau und ich während unseres Aufenthaltes in Tansania in den Schulen der Dörfer um den Kilimanjaro besuchten, und machten mich nachdenklich.

In Europa sieht man Kinder in fröhlicher Ausgelassenheit recht selten, der Anblick war eine wahre Freude, als Erwachsene haben wir es verlernt damit umzugehen und sind oft verklemmt. Viele junge Menschen fragen sich in Deutschland, kann man es noch verantworten und Kinder in diese Welt setzen? Obwohl mir die Frage eher als Vorwand denn als echte Sorge vorkommt, kann ich als alter Mann ein gewisses Verständnis dafür nicht unterdrücken. Die Koreaner scheinen sich um solche Gedanken nicht ernsthaft zu kümmern.

Das koreanische weiße Porzellan ist ohnehin seit meinen früheren Besuchen in Korea mein ausgesprochener Favorit gewesen, so konnte ich mit großer Freude die vielen Exponate genießen. Interessiert las ich den Hinweis, dass dieses Porzellan, das vornehme Ruhe ausstrahlt, bereits früher ausnehmend teuer und nur für zeremonielle Zwecke und die Aristokratie gedacht war, während das Volk billiges Geschirr aus dem haushaltsüblichen braunen Ton verwendete, wovon reichlich Museumsexponate aus der gleichen Periode zeugten. Entschädigt wurde ich auch durch die reichhaltige Sammlung der großartigen bemalten Seidenrollen mit den klassischen Blumenmuster, die sog. „4 Gentlemen" (= Pflaumenblüte, Orchidee, Chrysanthemen und Bambus), wobei gerade Bambus-Zeichnungen nicht nur wegen ihrer ausdrucksvollen Zartheit früher besonders beliebt waren. Bambus ist für den Asiaten eine romantische Pflanze, das Rauschen der Bambusblätter im Wind ist die richtige Untermalung für die zeitweise schweigsam-melancholische Stimmung Ostasiens. Die begleitende Erläuterung, dass die ostasiatische Kalligrafie als Versuch zu verstehen ist, aus dem „alten etwas Neues zu kreieren, ohne das Gesetz zu brechen“, fand ich recht typisch für das asiatische Verständnis. Ein guter Hinweis an unsere heutigen Künstler im Westen, in Anbetracht der Werke, die sie zustande bringen auf ihrer Suche nach Neuem!

Auf ihr neues Nationalmuseum sind die Koreaner nicht ohne Grund stolz. Der durchdachte Aufbau und die große Sammlung ostasiatischer Kunst sind zweifellos beeindruckend. Eine kleine Tonstatuette aus Khotan (2.-3. Jh.) habe ich darunter mit Vergnügen registriert, die eine Verbindung von Serapis mit Harpocrates (!) sein soll. Sollte es denn einen mir unbekannten griechischen Mediziner dieses Namens gegeben haben?

Einen Hinweis, den ich aus den im Museum angeführten Interpretationen der buddhistischen Periode entnommen habe, möchte ich hier kurz anführen, weil mir die Vorstellung originell und sympathisch erschien:

Nach dem buddhistischen Glauben soll die untere Welt aus Himmel, Erde, der Hölle der hungernden Geister, der Tierwelt und der Welt der Menschen bestehen. Im Museum sind Exponate aus einem buddhistischen Tempel aufgestellt und ein Schrein 
des Höllengottes Ksitigarbha wird erwähnt. Er sei jemand, der sich verpflichtet hat, in der Hölle zu bleiben, um die Geister der Hölle solange zu beschimpfen, bis es unter den Menschen „keine Leidenden mehr gibt“! So habe ich es zumindest verstanden. Ein bemerkenswerte Idee, die mir bis heute völlig entgangen war. Beim stillen Betrachten des Schreins kam mir flüchtig der Gedanke, ob es auch in der modernen medizinischen Welt, die teilweise einer Hölle gleicht, sinnvoll wäre, einen Ksitigarbha-Geist zu haben, der solange die Verantwortlichen peinigt, bis die Medizin sich wieder auf ihre Mission besinnt und sich wieder dort findet, wo sie ursprünglich war und wo ihre eigentliche Aufgabe liegt, nämlich im Dienst am kranken Menschen.

\section{Kurzer Ausblick \\ $\nabla$}

Die Reise nach Seoul war das Wiedersehen mit einer alten Kultur, die in die Postmoderne eingebrochen ist und beim Kampf mit der Globalisierung und ihren Folgen auf verlorenem Posten steht. Beim diesjährigen Weltkongress und all seinen Sitzungen, Zeremonien und sonstigen Abläufen gewann ich den Eindruck, dass die Medizin, so auch unser Fach, zur Zeit eine Übergangsperiode durchmacht, in der die derzeitigen Akteure sich selbst nicht im Klaren darüber sind, was man als echten Fortschritt aufnehmen, aufrecht erhalten und weiterentwickeln soll, und was eine Fehlentwicklung ist, die man am besten sofort unter den Tisch fallen lässt. Viele neue Medikamente, medizinische Apparate und Tech- niken, die relativ schnell auf den Markt kommen, werden auch schnell als „Pionierleistungen“ gepriesen, obwohl sie in der kurativen Praxis nach kritischer Abwägung nicht unverzichtbar, viele davon eher unnütz sind. Vergleichsweise sind die bisherigen Behandlungsmethoden in gleicher Weise wirksam, wenn man sie beherrscht, vielleicht etwas langsamer, dafür umso sicherer. Die Zulassung der meisten neuen, sogenannten „innovativen“ Medikamente wird offenbar mit Hilfe wohlwollender Gutachten von Wissenschaftlern, z.T. auch Ärzten, ermöglicht, die von den Industrievertretern geschickt mit „an Bord“ genommen wurden oder aus eigener Leichtfertigkeit dort hineingeraten sind.

Wenn man sich mit jüngeren Kollegen unterhält, die teilweise an verantwortlichen Gremien beteiligt sind oder dort maßgeblichen Einfluss haben, merkt man ihre Unsicherheit. Manche spüren ein eigenes Unbehagen über diese Zustände, was aber nichts daran ändert, dass sie dem herrschenden Trend widerspruchslos folgen und vieles als „Fortschritt“ preisen, was diesen Namen bei kritischer Abwägung nicht verdient. Auch erfahrene Fachvertreter sind mitunter von der Schnelligkeit neuer Entwicklungen und der massiven Werbung überwältigt, und verkünden ihre ehrliche Überzeugung, dass letzten Endes alles unserem Fach zugute käme. Das kritische, dringend notwendige Abwägen und Abfiltrieren des überbordenden Datenflusses scheinen sie der Einfachheit halber der Zukunft zu überlassen. So ist es sicherlich bequemer. Wenn man sich da nicht gewaltig täuscht...

Hinweis: Überarbeitete Fassung vom 21. 9. 2011 